\title{
POSITIONING OF THE PRECURSOR GAS INLET IN AN ATMOSPHERIC DIELECTRIC BARRIER REACTOR, AND ITS EFFECT ON THE QUALITY OF DEPOSITED TiO ${ }_{x}$ THIN FILM SURFACE
}

\author{
Jan PichaL*, Julia Klenko
}

\author{
Czech Technical University in Prague, Faculty of Electrical Engineering, Technicka 2, Prague 6, Czech Republic \\ * corresponding author: pichal@fel.cvut.cz
}

\begin{abstract}
Thin film technology has become pervasive in many applications in recent years, but it remains difficult to select the best deposition technique. A further consideration is that, due to ecological demands, we are forced to search for environmentally benign methods. One such method might be the application of cold plasmas, and there has already been a rapid growth in studies of cold plasma techniques. Plasma technologies operating at atmospheric pressure have been attracting increasing attention. The easiest way to obtain low temperature plasma at atmospheric pressure seems to be through atmospheric dielectric barrier discharge (ADBD). We used the plasma enhanced chemical vapour deposition (PECVD) method applying atmospheric dielectric barrier discharge (ADBD) plasma for $\mathrm{TiO}_{x}$ thin films deposition, employing titanium isopropoxide (TTIP) and oxygen as reactants, and argon as a working gas. ADBD was operated in filamentary mode. The films were deposited on glass. We studied the quality of the deposited $\mathrm{TiO}_{x}$ thin film surface for various precursor gas inlet positions in the ADBD reactor. The best thin films quality was achieved when the precursor gases were brought close to the substrate surface directly through the inlet placed in one of the electrodes. High hydrophilicity of the samples was proved by contact angle tests (CA). The film morphology was tested by atomic force microscopy (AFM). The thickness of the thin films varied in the range of $(80 \div 210) \mathrm{nm}$ in dependence on the composition of the reactor atmosphere. XPS analyses indicate that composition of the films is more like the composition of $\mathrm{TiO}_{x} \mathrm{C}_{y}$.
\end{abstract}

KEYwORDS: AFM, atmospheric dielectric barrier discharge, chemical composition, chemical vapour deposition, inlet position, precursor gas, surface quality, thin film, $\mathrm{TiO}_{x}, \mathrm{TiO}_{x} \mathrm{C}_{y}, \mathrm{XPS}$.

\section{INTRODUCTION}

Thin film deposition techniques and technologies have undergone serious development and cultivation in recent decades. A vast number of deposition methods are now available [6, 7], but it still remains arduous to select the best deposition method which is, at the same time, environment friendly. The application of cold plasmas sustaining under atmospheric pressure in combination with the chemical vapour deposition method seems to be a promising approach. Cold plasma is often produced in plasma jets, plasma torches and ADBD (for details about ADBD, see e.g. [5]). However, research in this area is still mostly restricted to the laboratory stage. For practical reasons, the most tested films are $\mathrm{SiO}_{x}$ and $\mathrm{TiO}_{x}$ coatings.

This paper summarizes results obtained when $\mathrm{TiO}_{x}$ films are deposited on glass substrates using the PECVD method in an ADBD plasma reactor when TTIP, (less toxic than $\mathrm{TiCl}_{4}$, which was used e.g. in [2]), is applied as a precursor, namely the connection between the precursor gas inlet position in the ADBD reactor and the deposited film quality. Some preliminary results have been published in 3,4 .

\section{EXPERIMENTAL}

\subsection{The Reactor and the experimental CONDITIONS}

Film deposition was performed with discharge power of about $350 \mathrm{~mW}[14 \mathrm{kV}, 50 \mathrm{~Hz}]$.

The experiments were carried out in an open flow-through type plasma reactor with dimensions $(90 \times 79 \times 41) \mathrm{mm}$. The scheme of the plasma reactor is shown in Fig. 1. Plasma sustained between two brass electrodes $[(45 \times 8 \times 18) \mathrm{mm}$ HV electrode, $(40 \times 17 \times 18) \mathrm{mm}$ ground electrode] placed within the reactor. A barrier manufactured from glass plate $((70 \times 46 \times 1) \mathrm{mm})$ covered the ground electrode. The distance between the electrodes was fixed at $4 \mathrm{~mm}$. Three types of HV electrode were used in the experiments, all of them with identical external dimensions, but they were differentiated by the hole (diameter $3 \mathrm{~mm}$ ) leading into the interelectrode region. The first electrode was without a hole, the hole of the second type was connected with one inlet (C) only, and the third type had two inlets, $\mathrm{C}$ and D (see Fig. 1. for simplicity all four inlets possitions are drawn here, although only one pair of inlets was used in each experiment).

ADBD sustained in filamentary mode. Thin films 


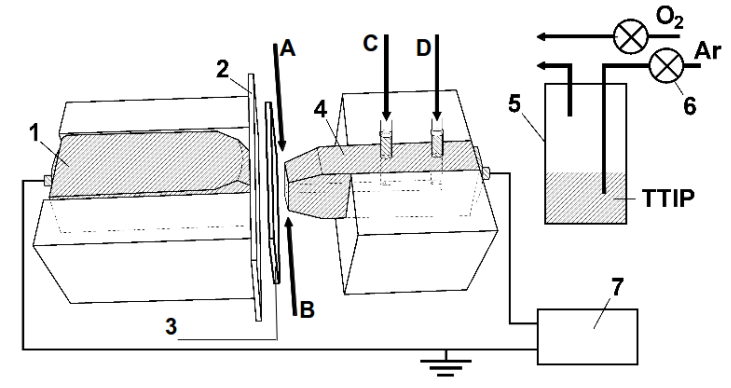

FiguRE 1. Scheme of the plasma reactor; 1 - ground electrode, 2 - dielectric barrier, 3 - substrate, 4 - HV electrode, 5 - evaporator, 6 - mass flow controller, 7 - HV supply, A, B, C, D - inlet positions.

were obtained at atmospheric pressure using titanium (IV) isopropoxide as a precursor (TTIP, $\mathrm{Ti}\left[\mathrm{OCH}\left(\mathrm{CH}_{2}\right)\right]_{4}$ and $97 \%$ purity).

TTIP volatilized at temperatures of $(30.0 \pm 0.5)^{\circ} \mathrm{C}$. It was mixed with argon in the evaporator, transported into the reactor and reacted with oxygen (or, in the first experiments, merely with dry air, when atmospheric oxygen took part in the reaction).

The gas flow rates were adjusted by means of the mass flow controllers. Deposition tests were performed with TTIP/Ar flows $(0.5 \div 4.0) \mathrm{slm}$. The oxygen/dry air flow was controlled within $(2.5 \div 10) \mathrm{slm}$.

The outer atmosphere was air with relative humidity of $(36 \div 47) \%$ and room temperature about $20^{\circ} \mathrm{C}$. The deposition time was 10 minutes in all experiments.

Unfortunately, the reactor has no "clean interface", so surface-related chemical reactions or contamination by ambient air species began while the films being removed from the reactor, before any test was initialized.

These chemical reactions also proceeded while the films were being stored. The deposited films were stored in darkness at room temperature $(20 \div 23){ }^{\circ} \mathrm{C}$, relative humidity $(30 \div 40) \%$, in plastic boxes in air at atmospheric pressure.

\subsection{Film ANALYSIS}

The surface morphology of the films was examined using the atomic force microscopy (AFM) technique in noncontact mode, performed under ambient conditions on an FRT AFM Scanning Probe Microscope at the Technical University of Liberec. All $(2 \times 2) \mu \mathrm{m}$ scans were processed by Gwyddion software for SPM (scanning probe microscopy) data visualization and analysis.

To analyse the hydrophilicity, a contact angle test (CA) was applied. CA was measured by a sessile drop technique when a constant time $(30 \mathrm{~s})$ passed after the dropping of distilled water about $0.5 \mu$ in volume. The CA of each sample was performed in 5 different positions at room temperature. The surface chem-

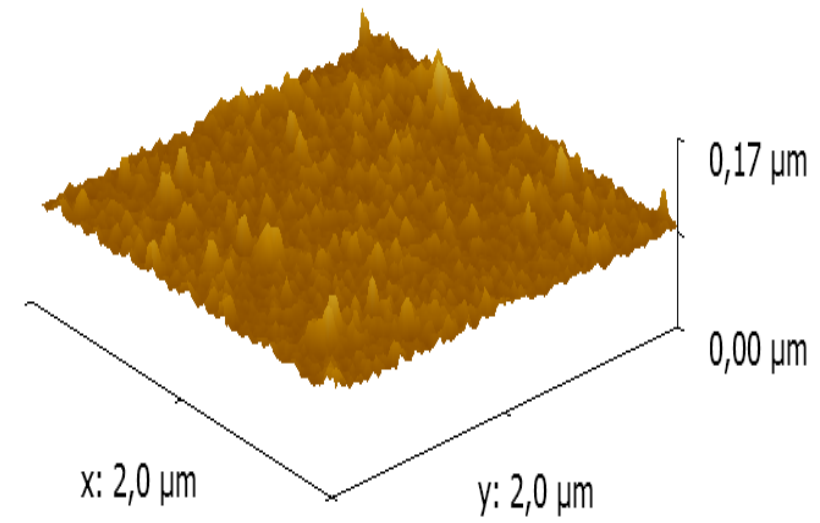

Figure 2. Topography of the $\mathrm{TiO}_{x}$ films; inlets: A (dry air from the atmosph.), C (TTIP/Ar, $0.5 \mathrm{slm}$ ).

ical composition of the films was investigated by X-ray photoelectron spectroscopy (XPS). A multi-channel hemispherical electrostatic analyser (Phobios 100, Specs) was used. The $\mathrm{Al} \mathrm{K} \alpha$ line $(1486.6 \mathrm{eV})$ was used with an X-ray incidence angle $45^{\circ}$ to the surface plane. The analyser was operated in retarding-field mode, applying pass energy of $40 \mathrm{eV}$ for the survey scans and $10 \mathrm{eV}$ for all core level data. The XPS peak positions were referenced at the aliphatic carbon component at $285.0 \mathrm{eV}$. Due to inadequate equipment we were unfortunately not able to clean the film profile by sputtering and perform XPS profile tests, so our research had to focus on film surface tests only.

\section{REsults}

Deposition of the films proceeds in substrate-surface limited reactions. The films were deposited in ADBD sustaining in filamentary mode.

The deposited surface was in general irregularly corrugated, hummocky, with growing thickness in spots where filaments bridged electrodes. Growing/diminishing thickness of the film area reflects local electric field inhomogeneities associated with the existence of filaments. The surface topography was similar to layers deposited with $\mathrm{TiCl}_{4}$ precursor [2, 10].

The gassing of the reactor was performed through various pairs of gas inlets (see Fig. 11). We used following combinations:

(1.) A (air at atm. pressure) and $\mathbf{B}$ (TTIP/Ar)

(2.) $\mathbf{A}$ (air at atm. pressure) and $\mathbf{C}$ (TTIP/Ar)

(3.) $\mathbf{C}$ (TTIP/Ar) and D (oxygen)

(4.) $\mathbf{C}$ (oxygen) and D (TTIP/Ar)

For each combination of inlets we tested the effect of TTIP and $\mathrm{O}_{2}$ in various concentrations in the TTIP $/ \mathrm{Ar} / \mathrm{O}_{2}$ mixture on the film characteristics.

Only the best results are mentioned in the following summary. 


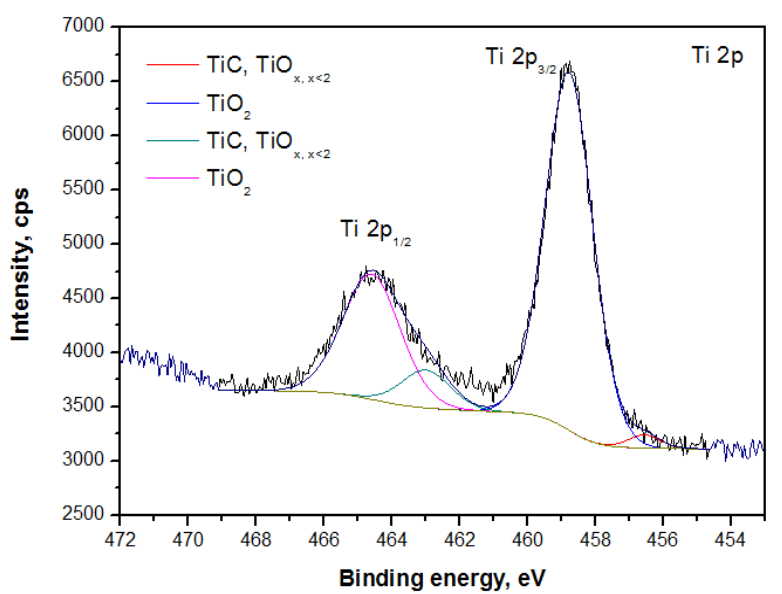

Figure 3. Ti 2p XPS spectrum of the film, inlets A, C.

1. The reactor was gassed both with $\mathrm{TTIP} / \mathrm{Ar}$ $(0.5 \mathrm{slm})$ and with dry air through inlets $\mathrm{A}$ and $\mathrm{B}$ in the walls of the reactor. Several differents positions of both inlets were tested, but only powder-like structures were deposited on the glass substrate for all used combinations of $\mathrm{A}$ and $\mathrm{B}$.

2. To reduce pulverization, the reactor was gassed through inlet $\mathrm{A}$ in the wall of the reactor with dry air, and TTIP/Ar $(0.5 \mathrm{slm})$ was fed through inlet C (the hole, $3 \mathrm{~mm}$ in diameter, in the $\mathrm{HV}$ electrode).

The film that formed on the glass substrate was very thin and was barely detectable. We suppose that the TTIP/oxygen reaction was weak, due to the low concentration of oxygen atoms (from the air) in the reactor atmosphere. Then the precursor molecules flew out from the inter-electrode space and later reacted with oxygen atoms (and we observed the $\mathrm{TiO}_{x}$ thin film deposited on the inner walls of the reactor).

AFM measurements revealed the existence of some salient parts on the uniform powder-like film surface (Fig. 2). Small hummocks were also visible.

$\mathrm{CA}$ measurements were almost impossible due to the inhomogeneity and the thinness of the film, and the results were not reproduciable. Only powderlike $\mathrm{TiO}_{x}$ structures were deposited on the substrate for TTIP/Ar flows higher than $0.5 \mathrm{slm}$.

The high-resolution XPS spectra for the main elements in the films are shown in Figs. 3 .5. Figure 3 represents the Ti $2 \mathrm{p}$ spectrum that consists of $2 \mathrm{p}_{3 / 2}$ and $2 \mathrm{p}_{1 / 2}$ spin orbit components located at $458.8 \mathrm{eV}$ and $464.6 \mathrm{eV}$, respectively. This position of the peak maxima indicates that the main titanium compound is $\mathrm{TiO}_{2}$. The small components on the lower binding energy side correspond to sub-stoichiometric titanium oxide $\mathrm{TiO}_{x}, x<2$.

The $\mathrm{O} 1 \mathrm{~s}$ spectrum is shown in Fig. 4. The peak consists of two distinct components at $530.5 \mathrm{eV}$ and $532.7 \mathrm{eV}$. The first component is classified as $\mathrm{TiO}_{2}$, whereas the second component corresponds to the carbon-oxygen species. The carbon-oxygen species are about three times more abundant than oxygen

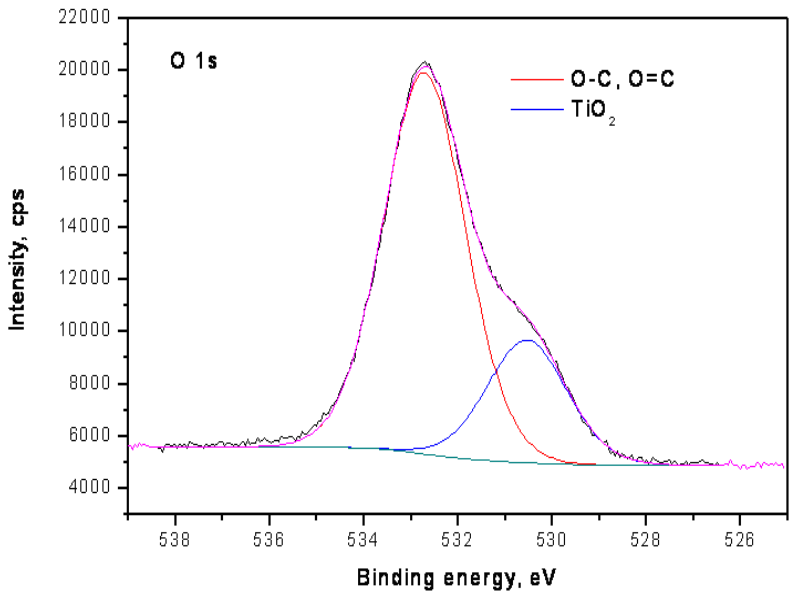

Figure 4. O 1s XPS spectrum of the film, inlets A, C.

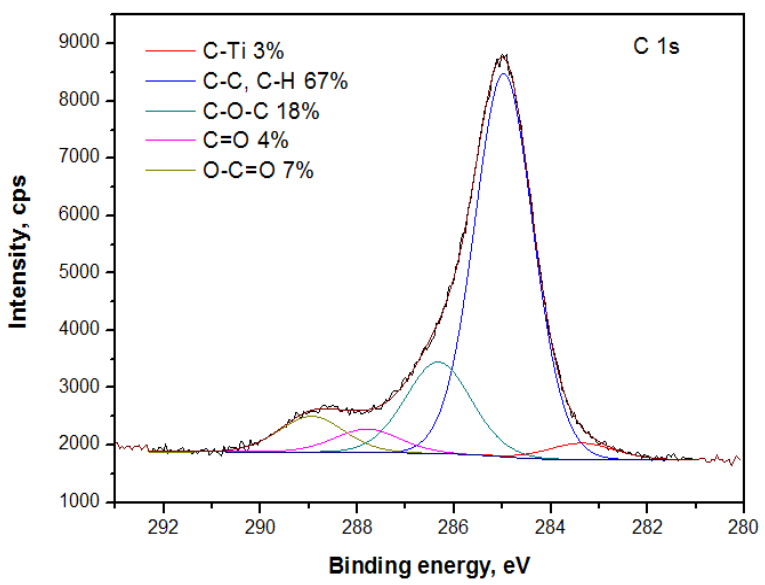

Figure 5. C 1s XPS spectrum of the film, inlets A, C.

bound in titanium (24\% against $76 \%$ ). We suppose that the carbon contamination was probably only superficial. This contamination may have originated both during the deposition process by impurities from the air and by post-discharge reactions and adsorption of various species from the ambient air atmosphere after the film removal from the reactor.

3. To improve the quality of the films and optimize the deposition conditions, both components were introduced through the inlets in the HV electrode, and the atmospheric air was replaced by oxygen (C (TTIP/Ar) and D (oxygen)).

Nevertheless problems still persisted. AFM analysis proved that the deposited films were not fully homogeneous (Fig. 6), and there were problems with creating the powder-like structures. For further details, see 3 .

4. The best quality films were deposited in the same adjustment, but unlike with the previous combination of inlets, oxygen $(2.5 \mathrm{slm})$ was fed through inlet $\mathrm{C}$ and TTIP/Ar mixture ( $1 \mathrm{slm}$ or $2 \mathrm{slm})$, i.e. the TTIP content (0.05 or 0.10$) \%$ was fed in through inlet D. The mixture entered through the hole (diameter $3 \mathrm{~mm}$ ) in the $\mathrm{HV}$ electrode into the inter-electrode space. 


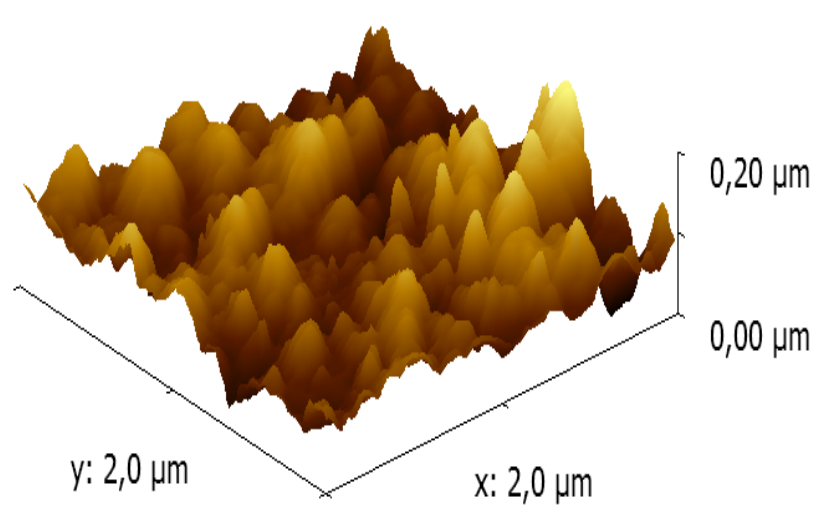

Figure 6. Inlets: C (TTIP/Ar, $1 \mathrm{slm})$, D $\left(\mathrm{O}_{2}, 2.5 \mathrm{slm}\right)$.

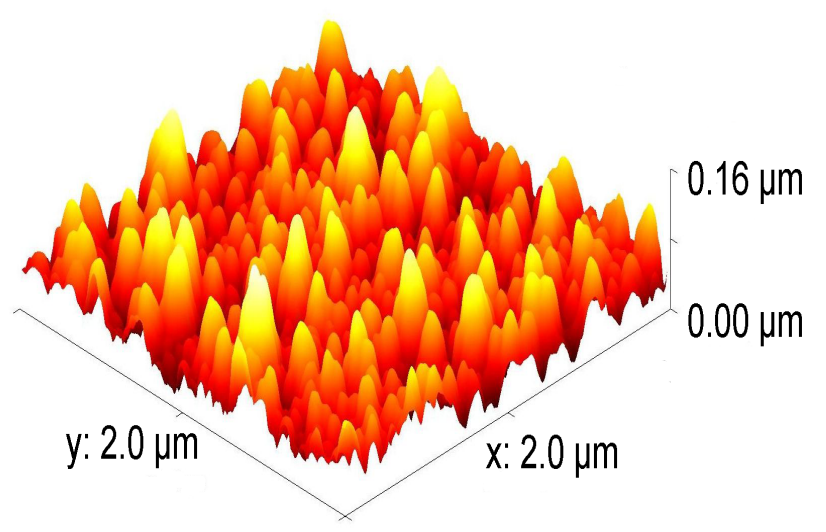

Figure 7. Topography of the $\mathrm{TiO}_{x}$ films; inlets: C (TTIP/Ar, $1 \mathrm{slm}), \mathrm{D}\left(\mathrm{O}_{2}, 2.5 \mathrm{slm}\right)$.

Figure 7 is AFM scan of the film surface deposited under optimum conditions. The surface is similar to the surface described in [10. It is characterised by higher hummocks than in Fig. 6, when film deposition in surface-limited reactions was accompanied by volume-limited dust generating reactions.

The CA tests proved that all samples were hydrophilic immediately after deposition. For an Ar/TTIP flow rate of $1 \mathrm{slm}$, the $\mathrm{CA}$ value attained $5^{\circ}$ immediately after deposition. The hydrophilicity of the films remained almost invariable in the first 7 days after deposition. Later, the wettability had worsened and within 28 days after deposition the $\mathrm{CA}$ value of all samples exceeded $40^{\circ}$.

Films deposited with TTIP/Ar flow 2 slm changed more rapidly from hydrophilic to hydrophobic.

The chemical composition of the films (TTIP/Ar flows $(0.5 \div 4.0 \mathrm{slm})$, oxygen $(2.5 \div 10)$ slm was almost constant. High-resolution spectra for the main elements in films are shown in Figs. 8 10.

The relatively high hydrocarbon contamination on the film surface was again most probably produced by the post-discharge reactions, and by the adsorption of various species from the ambient air atmosphere after the film was removed from the reactor.

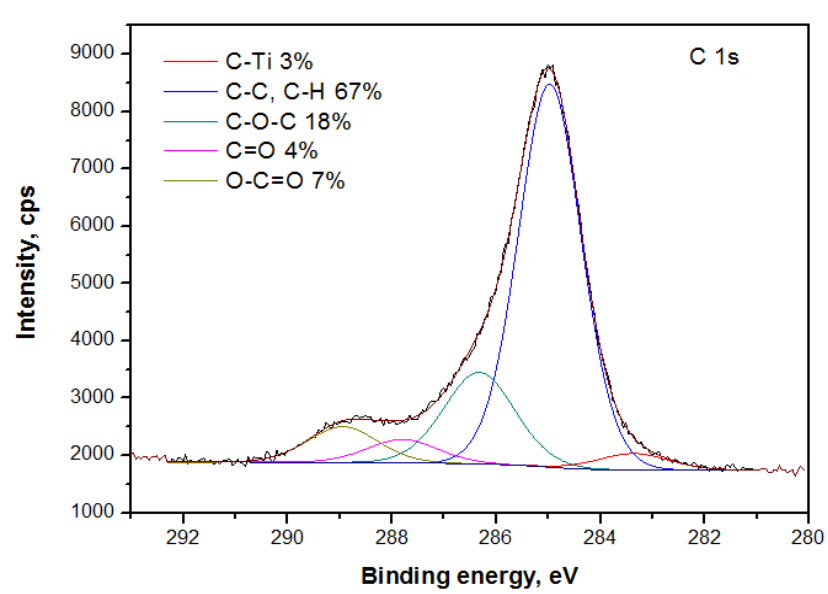

Figure 8. C 1s XPS spectrum of the film; inlets C (oxygen, $2.5 \mathrm{slm}$ ), D (TTIP/Ar, $2 \mathrm{slm}$ ).

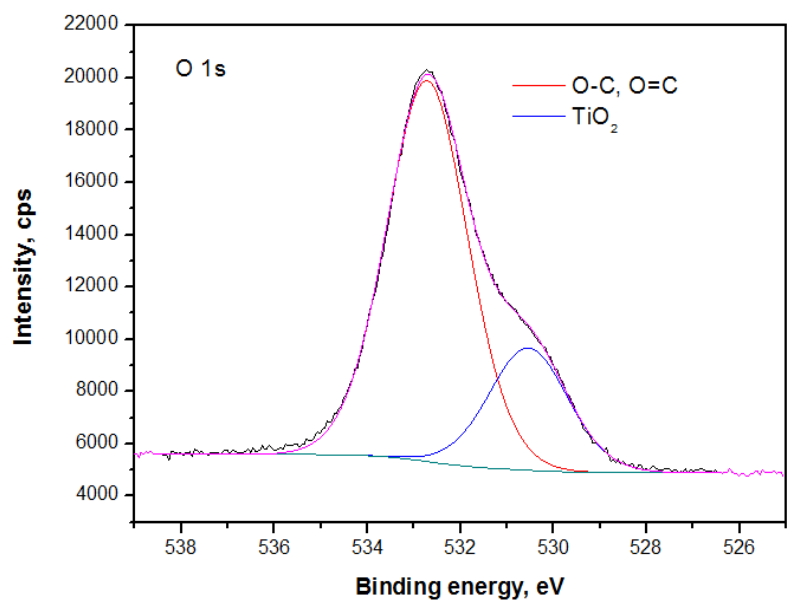

Figure 9. O 1s XPS spectrum of the film; inlets C (oxygen, $2.5 \mathrm{slm}$ ), D (TTIP/Ar, $2 \mathrm{slm})$.

The carbon (contamination) is partially bonded with titanium (the $\mathrm{C}-\mathrm{Ti}$ bond is about $283 \mathrm{eV}$ ). Carbon forms mostly $\mathrm{C}-\mathrm{C}(285 \mathrm{eV})$ backbone chains, some of which are partly oxidized (Fig. 8).

The $\mathrm{O}$ 1s spectra are shown in Fig. 9 . Note, that FWHM (full width at half of maximum) is more than $2 \mathrm{eV}$. This broadening is evidently caused by substoichiometric titanium oxides [1]. In addition, the second peak at $532.8 \mathrm{eV}$ can be considered as a contribution from single and double oxygen-carbon bonds $[9]$.

Titanium is a reactive element and easily forms oxides and carbides, which can be seen in the curve of Ti $2 p$ (Fig. 10p. The location of the strongest peaks at $458.8 \mathrm{eV}$ and $465 \mathrm{eV}$ indicates that the main titanium species is $\mathrm{TiO}_{2}[8$. The small peaks at $457 \mathrm{eV}$ and $462.6 \mathrm{eV}$ indicate mixed presence of substoichiometric titanium oxides $\mathrm{Ti}_{x} \mathrm{O}_{y}$ and titanium carbides TiC. The XPS spectra demonstrate that the titanium in the near surface area is strongly oxidized, the dominant species being $\mathrm{TiO}_{2}$ and substoichiometric titanium oxides. The deposition process more 


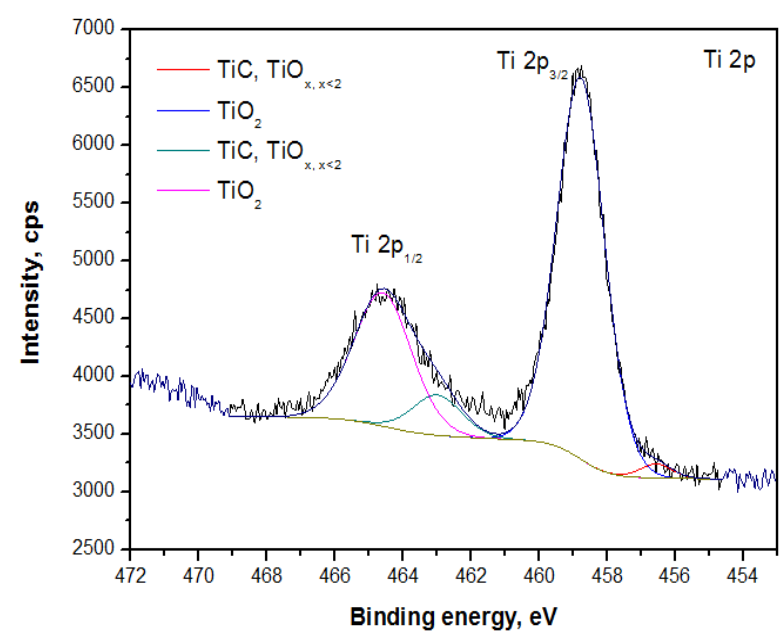

FIGURE 10. Ti 2p XPS spectrum of the film; inlets C (oxygen, $2.5 \mathrm{slm}$ ), D (TTIP/Ar, $2 \mathrm{slm})$.

likely produced $\mathrm{TiO}_{x} \mathrm{C}_{y}$ films instead of the primarily desired $\mathrm{TiO}_{x}$ films.

For more details see [4].

\section{Conclusion}

Film deposition on glass substrates was performed by the PECVD method in the ADBD plasma reactor. The plasma reactor was an open flow-through type. ADBD sustained in filamentary mode. The reactor atmosphere consisted either of TTIP/Ar/dry air or of a TTIP/Ar/oxygen mixture.

We studied the quality of the deposited $\mathrm{TiO}_{x}$ thin film surface for various precursor gas inlet positions in the ADBD reactor, and various precursor/oxidizer mixture compositions. The best film quality was achieved when the precursor and the oxydizer entered the discharge region instantly after they were mixed, through the hole adjacent to the substrate.

The surface topography was influenced by the nonequilibrium character of ADBD, leading to the irregularly corrugated and hummocky surface of the film.

CA tests proved the high hydrophilicity of the samples immediately after deposition. Later, the wettability of the films diminished, and the CA value of all samples exceeded 40 degrees after 28 days; the changes were probably related with chemical reactions between the surface of the film and the chemical groups involved in the air atmosphere.

XPS tests indicate that the deposition process more likely produced $\mathrm{TiO}_{x} \mathrm{C}_{y}$ films instead of the primarily desired $\mathrm{TiO}_{2}$ or $\mathrm{TiO}_{x}$ films. All samples exhibit contamination with carbon, probably caused by postdischarge reactions and by adsorption of various species from the ambient air atmosphere after the film was removed from the reactor.

Some problems of this deposition method are related with two different chemical processes that take place during deposition: surface-related chemical processes resulting in conventional PECVD film deposition, and undesired volume-related chemical processes resulting in dust production. The dust-producing mechanism prevails under certain working conditions (e.g. higher oxygen flow rate values). Dust particles, when created, remain in the discharge region and their layer(s) on the substrate hinder the effective formation of a more homogeneous film, and influence the quality of the film. Another problem of PECVD thin film deposition with ADBD seems to be with the filamentary character of the ADBD in some applications, leading to generation of hummocks that form a rough surface of the films.

\section{ACKNOWLEDGEMENTS}

This work was supported by Czech Technical University in Prague grant No. SGS10/266/OHK3/3T/13.

\section{REFERENCES}

[1] I. Bertoti, M. Mohai, J. L. Sullivan, S. O. Saied. Surface characterisation of plasma-nitrided titanium: an XPS study. Appl Surf Sci 84:357-371, 1995.

[2] Lan-Bo Di, Xiao-Song Li, Chuan Shi, et al. Atmospheric-pressure plasma CVD of $\mathrm{TiO} 2$ photocatalytic films using surface dielectric barrier discharge. J PhysD: Applied Physics 42:032001, 2009.

[3] Y. Klenko, J. Pichal. Deposition of TiO2 thin films using atmospheric dielectric barrier discharge. Problems of Atomic Science and Technology Series: Plasma Physics 48:177-179, 2008.

[4] Y. Klenko, J. Pichal. $\mathrm{TiO}_{x}$ films deposited by plasma enhanced chemical vapour deposition method in atmospheric dielectric barrier discharge plasma. Plasma Chemistry and Plasma Processing 32:1215-1225, 2012.

[5] U. Kogelschatz. Dielectric-barrier discharges: Their history, discharge physics and industrial applications. Plasma Chemistry and Plasma Processing 23:1-46, 2003.

[6] Seshan Krishna (ed.). Handbook of thin-film deposition. Elsevier, Oxford, 2012.

[7] D. M. Mattox. Handbook of Physical Vapor Deposition (PVD) Processing. Elsevier (William Andrew), Oxford, 2010.

[8] S. W. Ryu, E. J. Kim, S. K. Ko, S. H. Hahn. Effect of calcination on the structural and optical properties of $\mathrm{M} / \mathrm{TiO}_{2}$ thin films by rf magnetron co-sputtering. Materials Letters 58:582-587, 2004.

[9] T. Solomun, A. Schimanski, H. Sturm, E. Illenberger. Reactions of amide group with fluorine as revealed with surface analytics. Chemical Physics Letters 387:312-316, 2004.

[10] X. W. Zhang, G. R. Han. Microporous textured titanium dioxide films deposited at atmospheric pressure using dielectric barrier discharge assisted chemical vapor deposition. Thin Solid Films 516:6140-6144, 2008. 